\title{
BAG1 Overexpression Stabilizes High Molecular Tau Protein - a Crucial Role of the Co-chaperone in Tau Pathology
}

\author{
Sandra Caecilie Signore ${ }^{1, *}$, Fred Silvester Wouters ${ }^{2,3}$, Matthias Schmitz ${ }^{4}$, Mathias Baehr ${ }^{1}$, \\ Pawel Kermer ${ }^{1,5}$ \\ ${ }^{1}$ Department of Neurology, University Hospital Goettingen, Goettingen, Germany \\ ${ }^{2}$ Laboratory for Molecular and Cellular Systems, Department of Neuropathology, University Medical Center, Goettingen, Germany \\ ${ }^{3}$ DFG Research Center, Nanoscale Microscopy and Molecular Physiology of the Brain, Goettingen, Germany \\ ${ }^{4}$ Department of Neurology, University Medical Center Göttingen and the German Center for Neurodegenerative Diseases (DZNE), \\ Goettingen, Germany \\ ${ }^{5}$ Department of Neurology, Nordwest-Krankenhaus Sanderbusch, Sande, Germany
}

Email address:

ssignore@web.de (S. C. Signore)

${ }^{*}$ Corresponding author

\section{To cite this article:}

Sandra Caecilie Signore, Fred Silvester Wouters, Matthias Schmitz, Mathias Baehr, Pawel Kermer. BAG1 Overexpression Stabilizes High Molecular Tau Protein - a Crucial Role of the Co-chaperone in Tau Pathology. American Journal of Psychiatry and Neuroscience.

Vol. 9, No. 2, 2021, pp. 77-85. doi: 10.11648/j.ajpn.20210902.18

Received: June 2, 2021; Accepted: June 16, 2021; Published: June 23, 2021

\begin{abstract}
The Bcl-2-associated athanogene-1 (BAG1) exerts neuroprotective properties which has been shown in several studies of neurodegenerative disease models like Parkinson's disease, Huntington's disease and even cerebral ischemia. On the basis of the well-known neuroprotective function of the co-chaperone, we wanted to examine its properties in a model for Alzheimer's disease, a neurological disorder of great significance. One of the hallmarks of Alzheimer's disease, besides extracellular plaque formation, is the intra-neuronal accumulation of hyper-phosphorylated tau protein that leads to tau aggregation. When overexpressed together with a tau mutant with high propensity for aggregation, BAG1 led to the stabilization of high-molecular tau fragments in rat CSM 14.1 cells compared to wild-type cells. Deletion of the domain in BAG1 that is responsible for binding to Hsp70 (BAG $\Delta \mathrm{C}$ ) abolished this effect, which could be confirmed by immunocytochemistry. In fact, BAG1 does not only increase mutant tau aggregation but also prevents its degradation by the proteasome. Immunochemistry revealed that overexpression of the Bcl-2-associated athanogene-1 gives rise to large tau aggregates surrounded by lysosomes. Furthermore, toxicity assays indicated increased tau toxicity in BAG1 overexpressing cells. Hence, in contrast to other neurodegenerative diseases, BAG1 seems to enhance Alzheimer's pathology and to promote cell death due to the stabilization of aggregation-prone tau species that evade proteasomal clearance. To conclude, this analysis provides a new sight of the cochaperone BAG1 and yet again demonstrates its complex influence in a model of Alzheimer's disease.
\end{abstract}

Keywords: Alzheimer's Pathology, BAG1, Tau Protein, Co-chaperone, Neurodegeneration

\section{Introduction}

Bcl-2-associated athanogene-1 (BAG1) is the first identified member of the BAG family, a group of antiapoptotic proteins. This protein family currently consists of six members (BAG 1-6) sharing the BAG protein domain from which the name derives. With the exception of BAG-5, all BAG family members share the evolutionary highly conserved $\mathrm{BAG}$ domain near the $\mathrm{C}$ terminus $[1,2]$ promoting binding to the ATPase domain of Hsp70, thereby stimulating its ATPase activity [3-5]. BAG1 contains an N-terminal ubiquitin-like motif and was found to bind to the proteasome 
and to the E3 ligase carboxyl terminus of Hsc70-interacting protein (CHIP) [6, 7]. The function of BAG1 has been investigated in several disease models and its neuroprotectivity is of particular interest [8-13]. Furthermore, BAG1 is able to stimulate neuronal differentiation and fosters neuronal survival $[8,13]$. It has also been shown to be neuroprotective against stroke induced by middle cerebral artery occlusion in transgenic mice overexpressing BAG1 [14]. Moreover, the co-chaperone BAG1 speeds up differentiation when overexpressed in neurons [8]. One of its vital functions could be shown in experiments with transgenic mice lacking BAG1 which died during embryonic development as a result of apoptosis in the nervous system and liver [15]. In line with these findings, overexpression of BAG1 provides protection against various apoptotic insults $[11,14,16]$. In in vivo experiments it could be shown that BAG1 is able to enhance axonal regeneration in mice [17]. In models of Huntington's disease [18] and Parkinson's disease $[19,20]$, BAG1 proved to be neuroprotective.

In numerous reports BAG1 has been described to associate with Hsp70-Tau complex and to regulate proteasomal protein degradation [21]. Furthermore, BAG1-M has been shown to be up-regulated in the hippocampus of Alzheimer's disease patients indicating a crucial role of BAG1 in tau pathology [22].

In this study, we investigate the interaction of BAG1 and a specific mutant tau protein which has a high tendency for aggregation. The so-called 3PO-tau is a mutant form of fulllength tau protein with distinct amino acid changes [23]. It has been tested in different cell types and shown to form stable tau aggregates with highly phosphorylated tau protein, consequently representing one hallmark of Alzheimer's disease (AD) pathophysiology. In line with this, the disruption of the microtubule network and pronounced cellular toxicity can be found [23]. The 3PO-tau model is investigated thoroughly by solid-state NMR [24] as well as in vivo in rodent brains using viral injection [25], showing a strong AD-like impairment.

To our knowledge, the interaction of BAG1 with tau mutant isoforms with higher propensities for aggregation has not yet been examined. In this study, we characterize BAG1 as a tau-interacting protein that has the ability to stabilize high-molecular tau protein leading to huge intracellular aggregates and increased toxicity.

\section{Material and Methods}

\subsection{Generation of Mutant Tau Protein 3PO-tau}

The generation of different mutant tau proteins and 3POtau were described in detail by Illiev et al [23].

\subsection{Cell Culture}

CSM 14.1 cells were grown in Dulbecco's modified Eagle's medium supplemented with $10 \%$ fetal calf serum and penicillin/ streptomycin in $32^{\circ} \mathrm{C}$. Stably transfected CSMBAG1 were maintained in the same medium with an addition of puromycin $(8 \mathrm{lg} / \mathrm{mL})$.

SH-SY5Y cells (German Collection of Microorganisms and Cell Cultures, Germany) were grown in Dulbecco's modified Eagle's medium supplemented with $15 \%$ fetal calf serum and 10,000 IU penicillin/streptomycin at $37^{\circ} \mathrm{C}, 5 \%$ $\mathrm{CO} 2$ and $80 \%$ humidity. For generation of stable BAG1 overexpressing cell line, 50 to $70 \%$ confluent cells were transfected in six-well plates with Lipofectamine 2000 according to the supplier's protocol (Invitrogen, Carlsbad, USA) in serum-free medium containing $4 \mu \mathrm{g}$ of pFlag-BAG1 DNA [8] or pcDNA3 control vector (hereafter referred to as "empty vector"). After $24 \mathrm{~h}$, medium was replaced by regular growth medium. For transient transfections, Lipofectamine 2000 (Invitrogen, Carlsbad, CA, USA) was used.

\subsection{Protein Extract Preparation}

Cells were washed, scraped in ice-cold phosphate-buffered saline (PBS), and pelleted. Cell lysates were prepared using a buffer containing $50 \mathrm{mM}$ Tris- $\mathrm{HCl}, \mathrm{pH} 7.4,150 \mathrm{mM} \mathrm{NaCl}, 1 \%$ Triton X100, and complete protease inhibitor cocktail (Roche, Indianapolis, IN, USA) for $15 \mathrm{~min}$ at $4^{\circ} \mathrm{C}$, followed by $10 \mathrm{~min}$ centrifugation at $16000 \mathrm{x} \mathrm{g}$ at $4^{\circ} \mathrm{C}$. The pellets which were further used for the filter retardation assay were resuspended in the lysis buffer and sonicated. Unless stated otherwise, cells were analyzed $24 \mathrm{~h}$ post transfection. For Western Blotting, the loading volume was $15 \mu \mathrm{g}$ each.

\subsection{Filter Retardation Assay}

For the filter retardation assay, protein extracts were heated at $98^{\circ} \mathrm{C}$ for $3 \mathrm{~min}$ in $2 \%$ sodium dodecyl sulfate and $50 \mathrm{mM}$ dithiothreitol and filtered through a $0.2 \mu \mathrm{m}$ cellulose acetate membrane (Schleicher \& Schuell, Dassel, Germany) using a dotblot filtration unit. The membranes were further processed for immunodetection. The loading volume was $20 \mu \mathrm{g}$ for each sample.

\subsection{Immunofluorescence}

Cells cultured on coverslips were washed in PBS and fixed in PBS containing 4\% p-formaldehyde for $10 \mathrm{~min}$ at $37^{\circ} \mathrm{C}$, followed by three times 10-minute washing steps in PBS. Permeabilization was performed in $-20^{\circ} \mathrm{C}$ acetone for $10 \mathrm{~min}$ with subsequent pre-blocking in PBS containing 3\% bovine serum albumin. Cells were incubated in blocking solution containing primary antibody for $1 \mathrm{~h}$ at $37^{\circ} \mathrm{C}$. After washing three times in PBS-T (Tween) and incubation with Cy3conjugated secondary antibodies (dilution 1: 400; Jackson Immuno Research, West Grove, PA, USA) for $1 \mathrm{~h}$ at $37^{\circ} \mathrm{C}$, cells were again washed three times in PBS-T, stained with DAPI (4',6'-Diamidino-2-phenylindole), and mounted on microscope slides using moviol (Polysciences Inc., Warrington, PA, USA). Confocal images were acquired using an inverted microscope (DMIRE2; Leica, Wetzlar, Germany) equipped with a $63 \mathrm{x} / 1.4$ numerical aperture (NA) oil immersion objective. For Cy5 fluorescence, cells were excited with the $633 \mathrm{~nm} \mathrm{He-Ne} \mathrm{laser} \mathrm{line} \mathrm{and} \mathrm{emission} \mathrm{were}$ recorded between 650 and $750 \mathrm{~nm}$. Wide field fluorescent 
images were acquired using an Axioplan2 microscope (Zeiss, Göttingen, Germany) equipped with a $63 \mathrm{x} / 1.4$ NA oil objective.

\subsection{Antibodies}

The following antibodies were used at corresponding dilutions: rabbit polyclonal BAG 1680, 1: 1000 (Kermer et al. 2002), mouse monoclonal T46 Tau, 1: 1000 (Thermo Fisher Scientific, Camarillo, USA), mouse monoclonal PHF-AT8 tau 1:1000 (Thermo Fisher Scientific, Rockford, USA), 1:5000 mouse actin (Millipore).

Labeling was visualized by fluorochrome-conjugated secondary antibodies (anti-rabbit Cy3, Jackson Immuno Research Laboratories Inc., USA) or diaminobenzidine (DAB Vectastain, Vector Laboratories).

\subsection{Cell Survival Assays}

Cell survival was assessed by crystal violet staining. Cells were fixed with $1 \%$ glutaraldehyde, washed with phosphate-buffered saline (PBS), and stained with crystal violet $(0.02 \%$ in water). The amount of crystal violet bound to the cells was dissolved with $70 \%$ ethanol. Data collection was performed with a spectrophotometer at a wavelength of $550 \mathrm{~nm}$.

\section{Results}

\subsection{BAG1 Stabilizes High Molecular Tau Fragments and Enhances the Formation of Tau Aggregates}

CSM14.1 [26] neuronal cells were stably transfected with a plasmid encoding full-length BAG1 [8] driven by the cytomegalovirus promoter [2]. These stably BAG1expressing cells were transiently transfected with 3PO tau and wild-type tau.

Western blot analysis of cell lysates using the monoclonal antibody T46 for labeling tau protein demonstrated the expression of tau protein fragments $24 \mathrm{~h}$ after transfection in CSM14.1 wild-type cells. We detected a dominant band with a molecular weight of $95 \mathrm{kDa}$, and additional $43 \mathrm{kDa}$ and 26 $\mathrm{kDa}$ tau fragments. Note also the presence of bands with molecular weights exceeding $95 \mathrm{kDa}$, whose size cannot be accurately resolved but further support the occurrence of pronounced aggregation (Figure 1a).

Interestingly, in BAG1-overexpressing cells we found that tau protein in CSM 14.1 cells stably expressing BAG1 was fully stabilized in the $95 \mathrm{kDa}$ high molecular tau form (Figure 1b), with the smaller tau fragments seen in CSM14.1 wild-type cells completely lacking.

Addition of the proteasome blocker MG132 to CSM 14.1 wild-type cells prevented the formation of the small $43 \mathrm{kDa}$ and $26 \mathrm{kDa}$ fragments, but retained the high molecular weight $95 \mathrm{kDa}$ band, confirming the origin of the smaller bands as proteasomal degradation products of 3PO tau (Figure 1c). The same result was obtained in stably BAG1 overexpressing CSM14.1 cells.

\subsection{The Anti-C-terminal anti-tau T46 Antibody Specifically Labels Tau Protein}

The temporal expression pattern of 3PO-tau-GFP was examined using Western blot analysis after 3, 6, 9, 12, 15, 18, $21,24,48$ and 62 hours post transfection using the highly specific tau antibody T46 and an antibody against the GFP tag. As can be seen in Figure 2a, a steadily increasing amount of tau protein is generated with early formation (from 9 hours post transfection) of aggregated high molecular weight 95 $\mathrm{kDa}$ tau, followed by the generation of its proteolytic smaller fragments with $43 \mathrm{kDa}$ and $26 \mathrm{kDa}$ as shown by the detection of its C-terminal epitope using the T46 antibody (Figure 2A). The progression profile of the different tau forms can also be followed from the GFP detection bands. (Figure 2B).

\subsection{The Deletion Mutant BAGAC Is Not Capable of Stabilizing the High-Molecular Tau}

CSM14.1 cells were transiently transfected with the deletion mutant BAG $\Delta \mathrm{C}$ which is no longer capable of binding to Hsp70 [2]. In contrast to CSM14.1 cells expressing full-length Bag1, formation of the small $26 \mathrm{kDa}$ tau fragment could not be prevented, i.e., full stabilization of tau in the $95 \mathrm{kDa}$ high molecular weight form failed. As a consequence, the distribution of tau fragments was similar to that in CSM14.1 wild-type cells (Figure 3). Interestingly, the tau fragment of $43 \mathrm{kDa}$ that can be detected in CSM14.1 wild-type cells were not detectable.

\subsection{BAG1 Increases Mutant Tau Aggregation and Prevents Its Degradation by the Proteasome}

Tau aggregation was quantified by dot blot analysis of CSM 14.1 wild-type and stably BAG1-overexpressing CSM14.1 cells. Higher amounts of tau aggregation could be seen in BAG1-overexpressing as compared to wild-type cells (Figure 4a). Interestingly, increased tau aggregation goes along with increased phosphorylation as indicated by a substantial signal increase obtained with the phosphorylationspecific AT8 antibody (Figure 4b). Intriguingly, in BAG $\Delta \mathrm{C}$ transfected CSM14.1. cells, this effect is abolished (Figure $4 c)$.

\subsection{CSM14.1 BAG1-Overexpressing Cells Show Large Tau Aggregates Surrounded by Lysosomes}

Cytological examination of cells expressing 3PO-tau-GFP in absence and presence of stably expressed BAG1 at $48 \mathrm{~h}$ post 3PO-tau expression shows microtubule binding of GFP3PO-tau and the presence of punctate aggregates mainly around the nucleus (Figure 5a, arrow). In BAG1overexpressing cells, tau aggregates are larger but appear to still be concentrated around the nucleus (Figure 5b, arrow). These cells further develop amorphous tau aggregates which almost fill out the entire cell body (Figure 5b, arrowhead shows a large aggregate which presumable grew from microtubule-associated 3PO-tau). In BAG1-overexpressing cells, tau aggregates are surrounded by lysosomes (Figure 5c) 
underlining the potential role of lysosomes in tau metabolism.

\subsection{BAG1 Enhances 3PO-tau Cell Toxicity}

To examine cell viability, we performed cell death and cell survival assays. In BAG1-overexpressing cells, cell survival was decreased non-significantly $24 \mathrm{~h}$ and $48 \mathrm{~h}$ after transfection as detected by crystal violet assays (Figure 6a). Adding proteasome inhibitor restored cell survival in CSM WT and BAG1-overexpressing cells (Figure 6b).

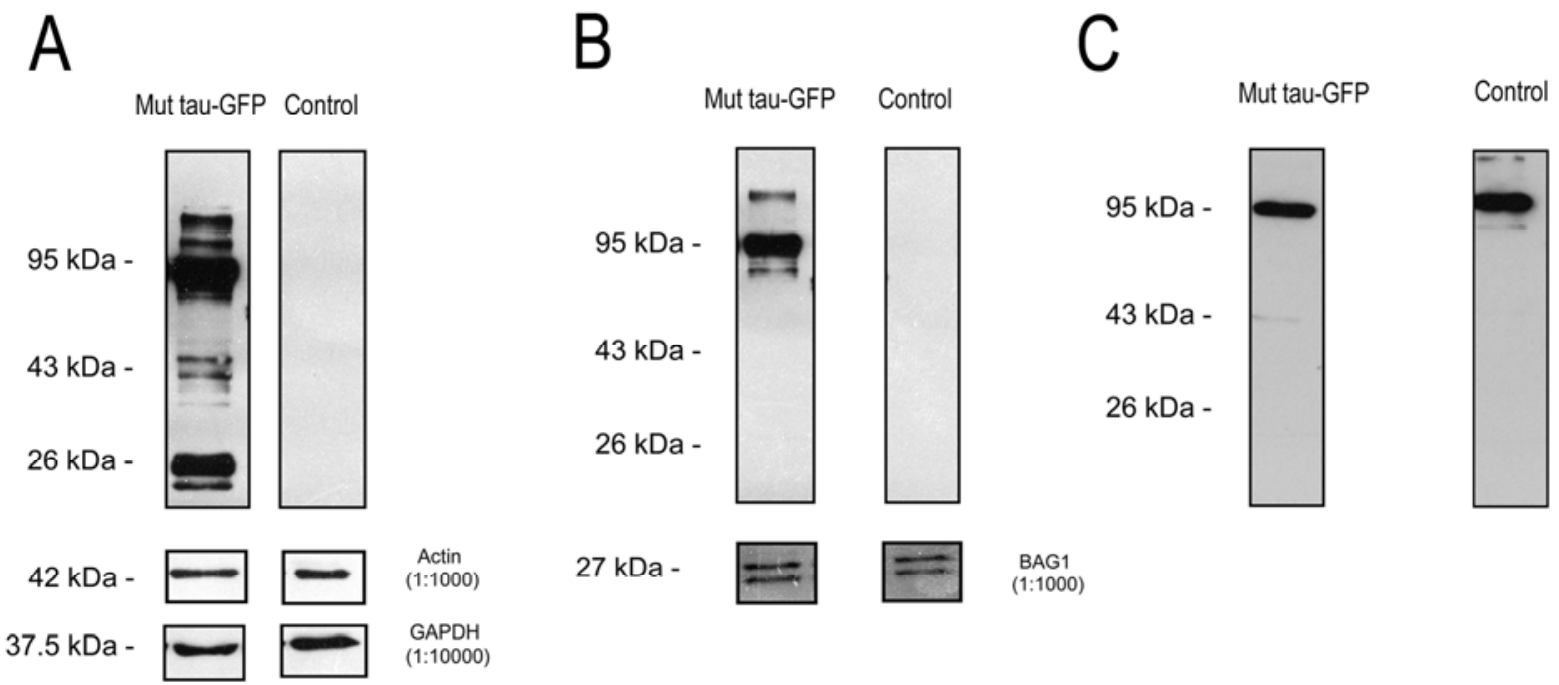

Figure 1. BAG1-overexpression stabilizes high-molecular tau.

a. CSM14.1 wild-type cells were analyzed 24h after transfection with the 3-PO tau mutant by western blotting using the anti-C-terminal anti-tau T46 antibody. Tau fragments could be detected in size of $95 \mathrm{kDa}, 43 \mathrm{kDa}$ and $26 \mathrm{kDa}$.

b. In contrast to that, Western blotting of transfected CSM14.1 BAG1-overexpressing cells with 3PO tau showed a stabilization of tau at $95 \mathrm{kDa}$ lacking smaller fragments.

c. Proteasomal inhibition by MG132 abolishes the effect of BAG1 on tau levels with detectable high-molecular tau levels but without medium-size or small tau fragments.

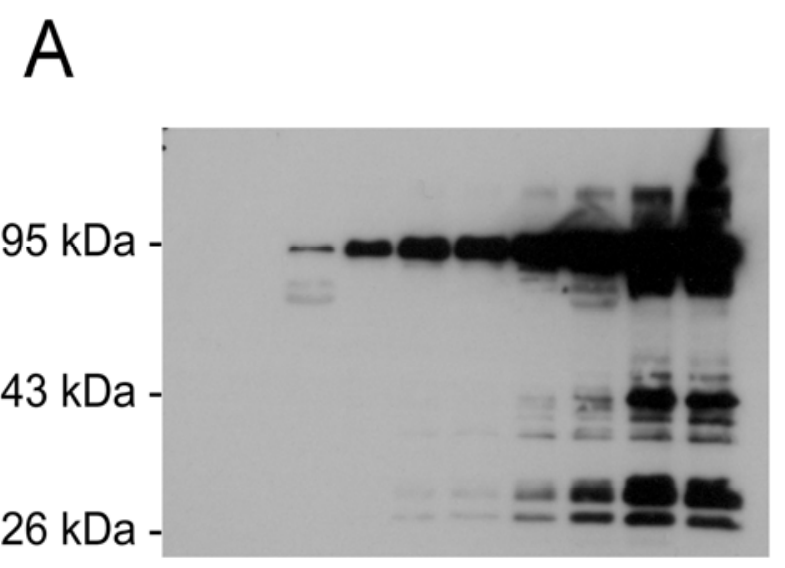

Hours: $3 \begin{array}{llllllll}3 & 9 & 1215 & 18 & 2124 & 48 & 62\end{array}$
B

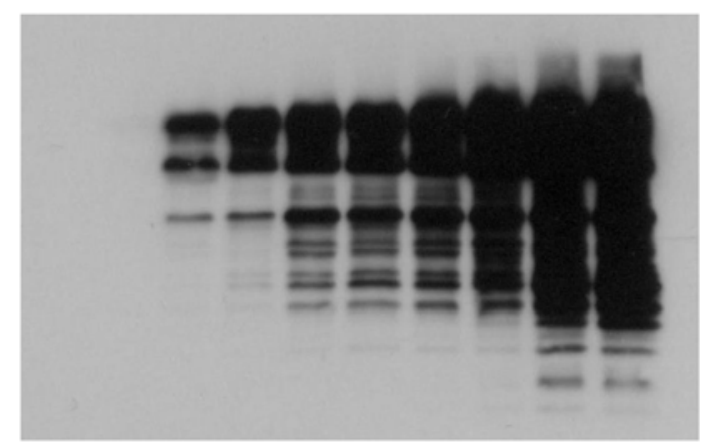

$\begin{array}{llllllllll}3 & 6 & 9 & 12 & 15 & 18 & 21 & 24 & 48 & 62\end{array}$

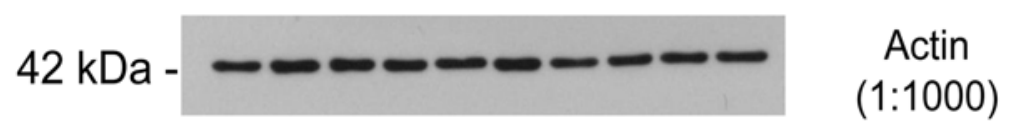

Figure 2. Anti-C-terminal anti-tau T46 antibody T46 antibody labels tau protein specifically.

Western Blot analysis analysis via timeline experiment after 3, 6, 9, 12, 15, 18, 21, 24, 48 and 62 hours. Increasing amount of tau protein can be detected with the highly specific tau antibody T46 labelling tau fragments of $26 \mathrm{kDa}, 43 \mathrm{kDa}$ and $95 \mathrm{kDa}$.

a. T46 antibody

b. GFP antibody 


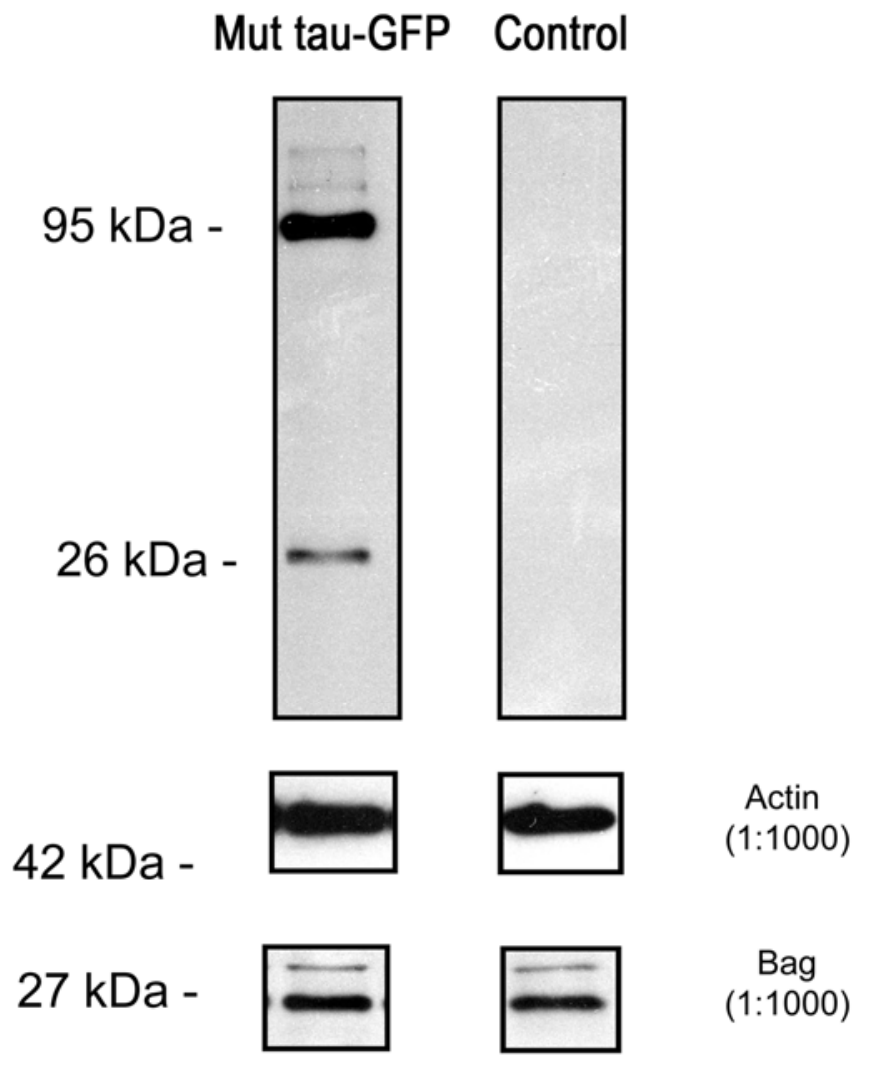

Figure 3. The deletion mutant BAGAC fails to fully stabilize tau in high-molecular protein form.

CSM14.1 wild-type cells transfected with the deletion mutant BAG $\Delta \mathrm{C}$ and the 3-PO tau mutant show similar results to CSM14.1 wild-type cells. Labelling with anti-C-terminal anti-tau T46 antibody, a molecular weight of $95 \mathrm{kDa}$ for tau protein and small tau fragments of $26 \mathrm{kDa}$ could be detected. Tau fragments of $43 \mathrm{kDa}$ were lacking in contrast to transfected CSM14.1 wild-type cells.

A
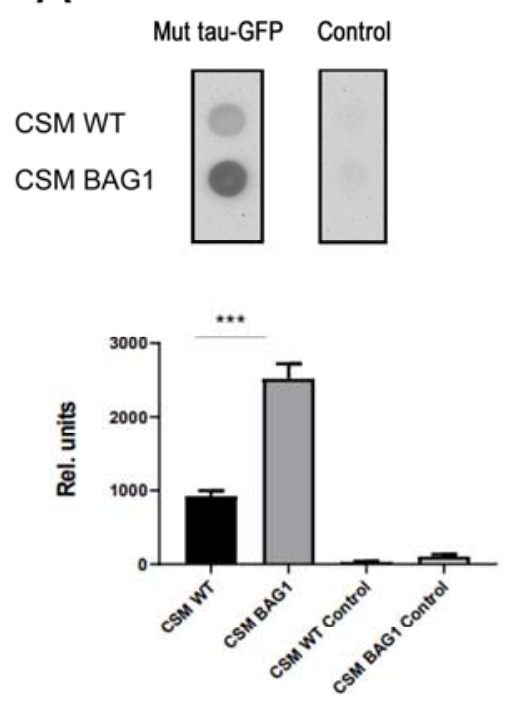

B
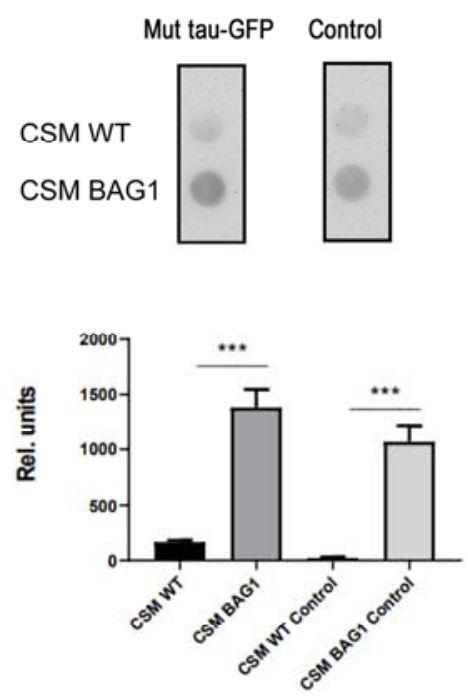

C
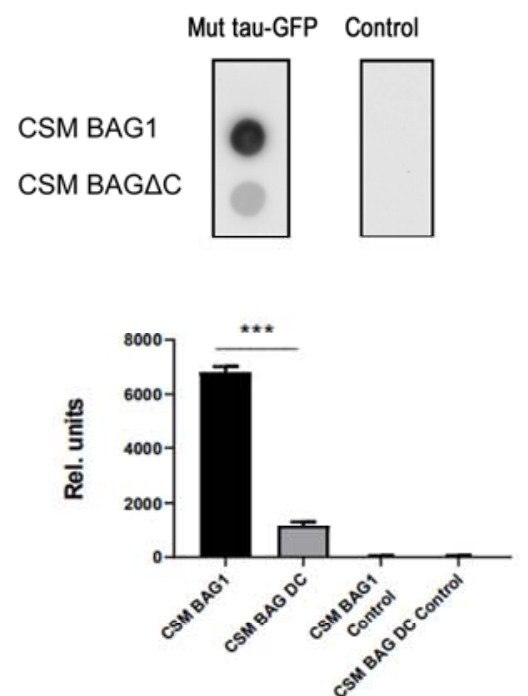

Figure 4. Dot Blot analysis reveals increased tau aggregation on BAG1-overexpressing cells in CSM 14.1 cells.

a. BAG1 overexpressing cells show a higher amount of tau aggregation (T46). Measurement of the dot intensity reveal a significant difference.

b. BAG1 overexpressing cells also show a higher phosphorylation (AT8). Measurement of the dot intensity reveal a significant difference.

c. In BAG $\Delta \mathrm{C}$ overexpressing cells, no higher amounts of tau aggregates (AT8) could be detectable.

All data represent mean values and SEM of three independent experiments (Student's t-test, ${ }^{*} \mathrm{p}<0.05 ;$ ANOVA, ${ }^{*} \mathrm{p}<0.05$ ) 
A
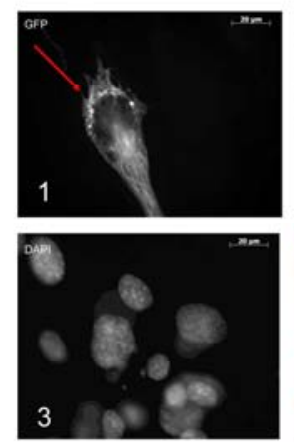

C

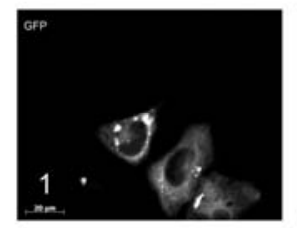

B
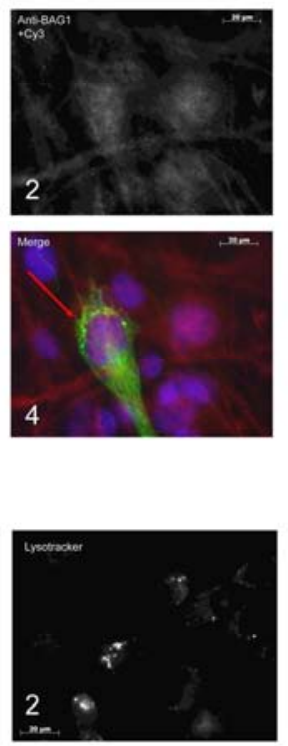
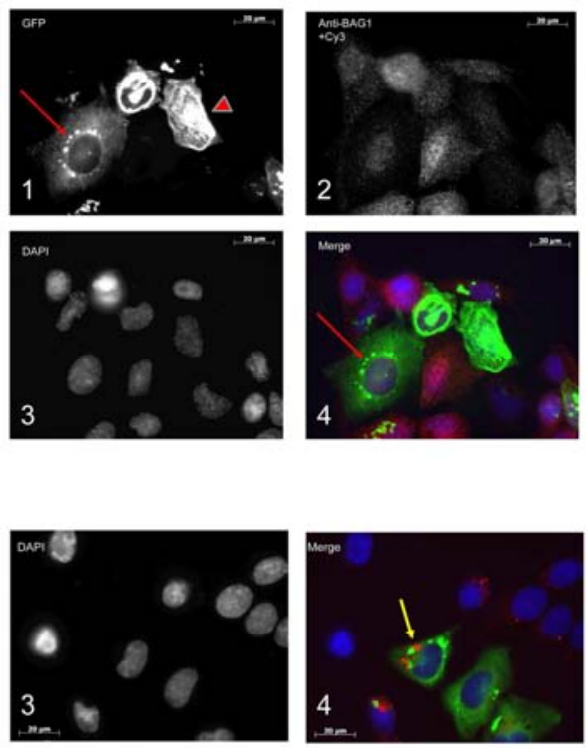

Figure 5. Cytology of CSM14.1 cells show aggregated tau formation after $48 \mathrm{~h}$ after transfection. Lysosomes surround tau aggregates in BAG1-overexpressing cells.

a. Wild-type cells show tau aggregates localized around the nucleus (arrow).

b. BAG1-overexpression lead to bigger tau aggregates still concentrated around the nucleus (arrow). Some cells are filled with amorphous tau aggregates which almost fill out the whole cell body (arrowhead). No colocalization of 3-PO-tau and BAG1 has been observed.

c. In BAG1-overexpressing cells, tau aggregates are surrounded by lysosomes but no colocalization has been found.

\section{A}

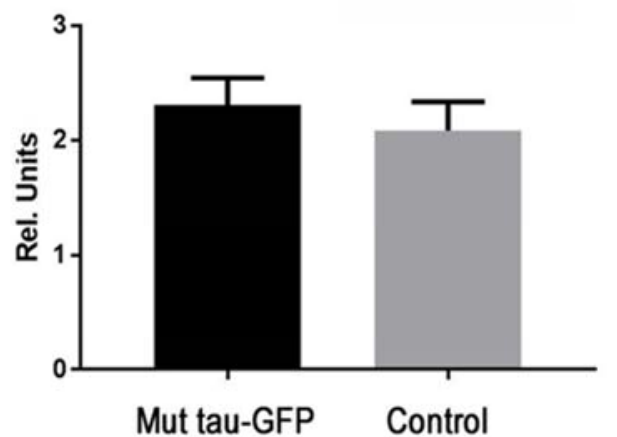

\section{B}

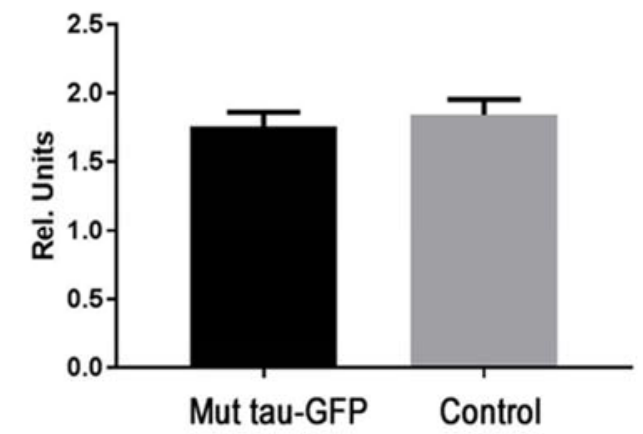

Figure 6. BAG1 does not prevent cell death due to 3PO-tau toxicity.

a. In crystal violet assays cell death was accelerated, albeit not statistically significant.

b. Adding proteasome inhibitor MG132 restored cell survival in CSM WT and BAG1-overexpressing cells.

All data represent mean values and SEM of three independent experiments (Student's t-test, *p $<0.05 ;$ ANOVA, ${ }^{*}$ p $<0.05$ )

\section{Discussion}

According to current literature, BAG1 plays a regulatory and protective role in neurodegenerative disorders in vitro as well as in vivo [8-14, 17-20]. BAG1 is also known to speed up neuronal differentiation [8]. We expected that BAG1 might have neuroprotective effects also in tau pathology with respect to clearance of tau aggregates, but our results showed the opposite: BAG1 facilitates mutant tau aggregation leading to enhanced hyperphosphorylation within the cells and resulting in higher toxicity.
Using 3PO tau, a tau mutant with a structurally optimized propensity for aggregation [23], it was possible to examine the effects of BAG1 in tau pathology under pathological conditions. As Liman et al. 2005 reported, the interaction of BAG1 and ATP-dependent Hsp70 mediates neuroprotection and increases chaperone activity [2], while the exact biochemical mechanism of BAG1/Hsp70 interaction remains unclear. Thus, not surprisingly, there are data indicating BAG1 as a stimulator of Hsp70 [2, 27, 28] while on the other hand there are data suggesting BAG1 having inhibitory effects on Hsp70 [2, 4, 29, 30]. 
We show that BAG1 stabilizes 3PO-tau in an aggregated, high molecular form, thereby increasing the toxicity of 3POtau expression. BAG $\Delta \mathrm{C}$, a deletion mutant that is no longer capable of binding to the C-terminus of Hsp70, does not exert such effects since BAG $\Delta \mathrm{C}$ cells transfected with the mutant tau isoform showed amounts of total tau protein and tau aggregation that are comparable to wild-type cells. Similarly, cell toxicity was comparable to wild type conditions, suggesting a protective effect in BAG $\Delta \mathrm{C}$ cells because of the prevention of interacting with Hsp70. Furthermore, our data shows that tau degradation to fragments of $42 \mathrm{kDa}$ and smaller is mediated through proteasomal cleavage and that the Hsp70 binding activity of BAG1 prevents efficient proteolysis. Intriguingly, the interaction of $\mathrm{Hsc} 70$ and $\mathrm{BAG}$ co-chaperones has been shown before [31].

In line with the observations made by Jinwal et al. [32] we could therefore confirm that the activity of Hsp70 appears to be crucial for tau metabolism. As BAG1 has been shown to increase total amount of high molecular tau protein leading to increased tau aggregation, it also increases 3PO-tau toxicity.

Using the selective proteasomal inhibitor MG132, a stabilization of high molecular tau protein in CSM14.1 cells was detectable. Small tau fragments of $26 \mathrm{kDa}$ in wild-type or BAG $\Delta \mathrm{C}$-transfected cells could no longer be seen indicating generation of small molecular tau fragments via another pathway than proteasomal degradation. It is known that lysosomes play an important role in tau degradation [33-36] and in our experiments the association of lysosomes around tau aggregates was clear. The signals did not colocalize, meaning that the aggregates themselves are not degraded by incorporation inside the lysosomes. This leaves the possibility of the release of sub-resolution fragments that are imported and degraded by a chaperone transport-dependent pathway.

BAG1 exerts no neuroprotection in our model of tau pathology, which is in contrast to the findings in other neurodegenerative diseases like Huntington's disease [18], Parkinson's disease [19, 20] and sporadic frontotemporal lobar degeneration [37]. Hence, BAG1 function seems to be more complex and diverse, and depending on diseasespecific interaction partners.

Like similar observations by other scientists [21, 22] our findings are of clinical relevance as they show that the current focus on chaperone function in the etiology and diagnosis of Alzheimer's disease should be broadened to also include co-chaperones. Elliott et al. pointed out that the cochaperone BAG1M is up-regulated in the hippocampus of $\mathrm{AD}$ patients and associates with tau protein [22]. Interestingly, BAG1M also showed an interaction with amyloid precursor protein (APP) indicating an important role for the co-chaperone in Alzheimer's disease pathology. The in vivo expression and effect of BAG1 in different stages of Alzheimer's disease could be of particular interest. Maybe an overexpression of BAG1 could serve as a potential biomarker for progressive Alzheimer's disease. For further investigation, testing of liquor samples of patients with different stages of Alzheimer's disease including patients with Mild Cognitive Impairment (MCI) need to be done. Due to the lack of biomarkers, these studies are of great relevance.

These ideas are inspired by the remarkable findings of the last years when BAG1 was described as a switch control in certain oncological diseases like acute myeloid leukemia [38]. In breast cancer, BAG1 was even identified to have significantly prognostic value [39]. The interaction of BAG1S, a BAG1 isoform, with the HSP70 chaperone complex selectively mediates cell survival in MYC overexpressing osteosarcoma cells. The authors of this publication stated the high importance due to the fact that most human cancers show a MYC overexpression indicating a possible treatment opportunity [40].

\section{Conclusion}

We showed that the co-chaperone BAG1 accelerates tau pathology in a model of Alzheimer's disease. This role is quite unique compared to its neuroprotective role in other neurological disorders. In conclusion, several studies showed that the co-chaperone it is one of the most important players and is characterized as protective or deteriorating via mediation of crucial intracellular pathways. Thinking of the outcome of individual patients, BAG1 overexpression could be crucial when it comes to disease progression. Many questions arise: Is BAG1 a biomarker in diagnosis or even in treatment sequence? Can a treatment against or for BAG1 be used in certain diseases?

Taken the studies concerning BAG1 and its interaction with Hsp70 into account, we recommend further investigations in neurological and oncological diseases in vitro and in vivo for potential biomarker relevance and thus for possible treatment options.

\section{Declarations}

\section{Funding Statement}

This study was supported by Deutsche Forschungsgemeinschaft (DFG) Research Center for Molecular Physiology of the Brain. Project title: BAG-1 in neurodegenerative disease.

\section{Conflict of Interest}

The authors declare that they have no competing interests.

\section{Author Contributions}

All authors contributed to the study conception and design. Material preparation, data collection and analysis were performed by SC Signore. The first draft of the manuscript was written by SC Signore and all authors commented on previous versions of the manuscript. All authors read and approved the final manuscript.

\section{Availability of data And Material}

The authors declare that the data supporting the findings of this study are available within the article. 


\section{Compliance with Ethical Standards}

This article does not contain any studies with human or animal subjects.

\section{Consent to Participate}

Not applicable since this study does not involve research on human subjects.

\section{Consent for Publication}

Not applicable since this study does not involve research on human subjects.

\section{Acknowledgements}

The study was performed within the University Medical Center Goettingen in the laboratory of Professor Baehr, M. D., Head of Department of Neurology.

\section{References}

[1] Takayama S and Reed JC. Molecular chaperone targeting and regulation by BAG family proteins. Nat Cell Biol. 2001; 3: E237-E241.

[2] Liman $\mathbf{J}$ et al. Interaction of BAG1 and Hsp70 mediates neuroprotectivity and increases chaperone activity. Mol Cell Biol 2005; 25, 3715-3725.

[3] Hohfeld J and Jentsch S. GrpE-like regulation of the hsc70 chaperone by the anti-apoptotic protein BAG1. EMBO J 1997; $16,6209-6216$.

[4] Takayama $\mathrm{S}$ et al. BAG1 modulates the chaperone activity of Hsp70/Hsc70. EMBO J 1997; 16, 4887-4896.

[5] Sondermann $\mathrm{H}$ et al. Structure of a Bag/Hsc70 complex: convergent functional evolution of Hsp70 nucleotide exchange factors. Science 2001; 291: 1553-1557. doi: 10.1126/science. 1057268 .

[6] Luders J, Demand J and Hohfeld J. The ubiquitin-related BAG1 provides a link between the molecular chaperones Hsc70/Hsp70 and the proteasome. J Biol. Chem 2000; 275 4613-4617.

[7] Demand J, Alberti S, Patterson and Hohfeld J. Cooperation of a ubiquitin domain protein and an E3 ubiquitin ligase during chaperone/proteasome coupling. Curr Biol 2001; 11, 15691577 .

[8] Kermer P et al. BAG1 is a regulator and marker of neuronal differentiation. Cell Death Differ 2002; 9: 405-413.

[9] Schulz JB, Bremen D, Reed JC et al. Cooperative interception of neuronal apoptosis by BCL-2 and BAG1 expression: prevention of caspase activation and reduced production of reactive oxygen species. J Neurochem 1997; 69: 2075-2086.

[10] Takaoka A et al. Anti-cell death activity promotes pulmonary metastasis of melanoma cells. Oncogene 1997; 14: 2971-2977.

[11] Takayama $\mathrm{S}$ et al. Cloning and functional analysis of BAG1: a novel Bcl-2binding protein with anti-cell death activity. Cell 1995; 80: 279-284.
[12] Townsend PA et al. BAG1 proteins protect cardiac myocytes from simulated ischemia/reperfusion-induced apoptosis via an alternate mechanism of cell survival independent of the proteasome. J Biol Chem 2004; 279: 20723-20728.

[13] Liman J et al. Subcellular distribution affects BAG1 function. Brain Res 2008; 1198: 21-26.

[14] Kermer P et al. 2003. BAG1 over-expression in brain protects against stroke. Brain Pathol 2003; 13: 495-506.

[15] Götz R et al. Bag1 is essential for differentiation and survival of hematopoietic and neuronal cells. Nat Neurosci 2005; 8: $1169-1178$

[16] Townsend, PA et al. BAG1 prevents stress-induced long-term growth inhibition in breast cancer cells via a chaperonedependent pathway. Cancer Res 2003; 63: 4150-4157.

[17] Planchamp V et al. 2008. BAG1 promotes axonal outgrowth and regeneration in vivo via Raf- 1 and reduction of ROCK activity. Brain 2008; 131: 2606-2619.

[18] Sroka $\mathrm{K}$ et al. BAG1 modulates huntingtin toxicity, aggregation, degradation, and subcellular distribution. $J$ Neurochem 2009; 111 (3): 801-7.

[19] Deeg $\mathrm{S}$ et al. BAG1 restores formation of functional DJ-1 L166P dimers and DJ-1 chaperone activity. J Cell Biol 2010; 188 (4): 505-13.

[20] Kermer P et al. BAG1 is neuroprotective in in vivo and in vitro models of Parkinson's disease. J Mol Neurosci 2015; 55 (3): 587-95.

[21] Elliott E, Tsvetkov P and Ginzburg I. BAG1 associates with Hsc70. Tau complex and regulates the proteasomal degradation of Tau protein. J Biol Chem 2007 Dec 21; 282 (51): 37276-84.

[22] Elliott E, Laufer O and Ginzburg I. BAG1M is up-regulated in hippocampus of Alzheimer's disease patients and associates with tau and APP proteins. J Neurochem 2009; 109 (4): 116878 .

[23] Iliev AI et al. Removal of pattern-breaking sequences in microtubule binding repeats produces instantaneous tau aggregation and toxicity. J Biol Chem 2006; 281 (48): 37195204.

[24] Daebel V et al. Beta-Sheet core of tau paired helical filaments revealed by solid-state NMR. J Am Chem Soc 2012; 134, 13982-13989.

[25] Dassie E et al. Focal expression of adeno-associated viralmutant tau induces widespread impairment in an APP mouse model. Neurobiol Aging 2013; 34, 1355-1368.

[26] Zhong LT et al. Bcl-2 inhibits death of central neural cells induced by multiple agents. Proc Nat Acad Sci 1993. USA 90: 4533-4537.

[27] Gassler CS et al. BAG1M accelerates nucleotide release for human Hsc70 and Hsp70 and can act concentration-dependent as positive and negative cofactor. $J$ Biol. Chem 2001; 276: 32538-32544.

[28] Terada K and Mori M. Human DnaJ homologs dj2 and dj3, and BAG1 are positive cochaperones of hsc70. J Biol Chem 2000; 275: 24728-24734. 
[29] Gebauer, M., M. Zeiner, and U. Gehring. 1997. Proteins interacting with the molecular chaperone hsp70/hsc70: physical associations and effects on refolding activity. FEBS Lett. 417: 109-113.

[30] Zeiner M, Gebauer M and Gehring U. Mammalian protein RAP46: an interaction partner and modulator of $70 \mathrm{kDa}$ heat shock proteins. EMBO J 1997; 16: 5483-5490.

[31] Rauch JN, Zuiderweg ER and Gestwicki JE. Non-canonical Interactions between Heat Shock Cognate Protein 70 (Hsc70) and Bcl2-associated Anthanogene (BAG) Co-Chaperones Are Important for Client Release. J Biol Chem 2016; 291 (38): 19848-57.

[32] Jinwal UK et al. Chemical manipulation of hsp70 ATPase activity regulates tau stability. $J$ Neurosci. 2009 Sep 30; 29 (39): 12079-88.

[33] Bednarski E and Lynch G. Cytosolic proteolysis of tau by cathepsin $\mathrm{D}$ in hippocampus following suppression of cathepsins B and L. J Neurochem 1996; 67 (5): 1846-55.

[34] Bi X et al. Novel cathepsin D inhibitors block the formation of hyperphosphorylated tau fragments in hippocampus. $J$ Neurochem 2000; 74 (4): 1469-77.
[35] Wang $\mathrm{Y}$ et al. Tau fragmentation, aggregation and clearance: the dual role of lysosomal processing. Hum Mol Genet 2009; Nov 1; 18 (21): 4153-70.

[36] Arnaud LT, Myeku N and Figueiredo-Pereira ME. Proteasome-caspase-cathepsin sequence leading to tau pathology induced by prostaglandin $\mathrm{J} 2$ in neuronal cells. $J$ Neurochem 2009; 110 (1): 328-42.

[37] Venturelli E et al. BAG1 is a protective factor for sporadic frontotemporal lobar degeneration but not for Alzheimer's disease. J Alzheimers Dis 2011; 23 (4): 701-7.

[38] Aveic S, Pigazzi M, Basso G. BAG1: The Guardian of AntiApoptotic Proteins in Acute Myeloid Leukemia. PLoS One 2011; 6 (10): e26097.

[39] $\mathrm{Du} \mathrm{JX}$ et al. Establishment and validation of a novel autophagy-related gene signature for patients with breast cancer. Gene 2020; 15; 762: 144974.

[40] Gennaro VJ, Wedegartner H and McMahon SB. Interaction between the BAG1S isoform and HSP70 mediates the stability of anti-apoptotic proteins and the survival of osteosarcoma cells expressing oncogenic MYC. BMC Cancer 2019; 22; 19 (1): 258 . 